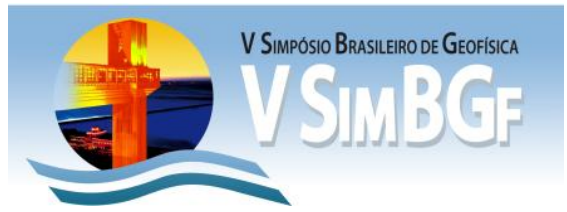

\title{
Medição e correção de fase em reflexões acima do ângulo crítico.
}

\author{
Allan Segovia Spadini*1 ${ }^{*}$ Liliana Alcazar Diogo ${ }^{1}$
}

1'Instituto de Astronomia, Geofísica e Ciências Atmosféricas - Universidade de São Paulo

Copyright 2012, SBGf - Sociedade Brasileira de Geofísica

Este texto foi preparado para a apresentação no V Simpósio Brasileiro de Geofísica Salvador, 27 a 29 de novembro de 2012. Seu conteúdo foi revisado pelo Comite Técnico do V SimBGf, mas não necessariamente representa a opinião da SBGf ou de seus associados. É proibida a reprodução total ou parcial deste material para propósitos comerciais sem prévia autorização da SBGf.

\begin{abstract}
In this work a correction to the phase shift observed in wavelets of reflections over the critical angle is evaluated. To measure the correct phase shift two different methods, rotation by maximization of the kurtosis and measurement of the instantaneous phase at the position of maximum value of the envelope, were tested. The objectives of this study was allow the usage of longer offset ranges, improving stacking, and also making possible to measure the phase correction that needs to be applied to each trace. The results of application of the two different methods were tested over synthetic and real data. In synthetic data it was possible to compare the phase that generated the data and improvements were observed in the stacking of the rotated data. And in real data an improvement of the interpretability of the section was observed.
\end{abstract}

\section{Introdução}

$\mathrm{Na}$ sísmica de reflexão rasa, com alvos de profundidade até 100 metros, frequentemente ocorrem situações em que são utilizados afastamentos fonte-receptor longos em relação à profundidade dos refletores, para que alguns tipos de ruídos coerentes sejam evitados e também para tirar vantagem do fato das reflexões em afastamentos longos serem mais fortes. Entretanto, a utilização de afastamentos longos traz outros desafios para o processamento dos dados: i) a aproximação hiperbólica para a equação de tempo de trânsito, convencionalmente utilizada nos procedimentos de análise de velocidades, não é válida, resultando na determinação de velocidades erradas; ii) o estiramento do pulso devido a correção de normal moveout (NMO) é acentuado, onde o procedimento usual é o silenciamento dos sinais; iii) mudança na fase do pulso refletido acima do ângulo crítico de incidência.

Os três aspectos mencionados acima são considerados neste trabalho. Contudo, o principal alvo de estudo desta pesquisa é a identificação e correção das mudanças de fase nos pulsos refletidos.

O fenômeno de mudança na fase das wavelets para reflexões acima do ângulo crítico foi discutido em detalhe nos trabalhos de Arons \& Yennie (1950) e Aki \& Richards (1980) e foi observado nos trabalhos de Pullan \& Hunter (1995) e Diogo, Diagon \& Prado (2004). Sendo que nestes trabalhos a mudança na fase prevista e/ou observada é independente da frequência, ou seja, pode ser corrigida através de uma rotação constante na fase do traço sísmico.
Uma forma de correção da fase do traço sísmico através da maximização do kurtosis foi mostrada por Levy \& Oldenburg (1987), Longbottom et al. (1988) e White (1988). O objetivo da metodologia empregada nestes trabalhos foi reduzir os graus de incerteza encontrados na deconvolução por mínima entropia de Wiggins (1978). Desta forma o método de rotação por fase constante do traço sísmico foi empregado após o procedimento de deconvolução por fase mínima ou divisão do espectro de amplitude no domínio da frequência, corrigindo a fase residual observada. Tanto a deconvolução por mínima entropia quanto o método de rotação de fase constante são baseados na maximização do kurtosis para que o traço resultante possua um valor mínimo de entropia. Considerando o trabalho de Berkhout (1977), onde é dada uma equação para o comprimento efetivo da wavelet, uma wavelet de fase zero possui menor comprimento e maior amplitude. Sendo assim, a correção por fase constante poderia melhorar o empilhamento e a interpretação das seções. Além disso, seria possível medir a correção aplicada para levar a wavelet para fase zero, possibilitando estudar melhor o efeito da mudança de fase em reflexões após o ângulo crítico.

No trabalho de Bodine (1984), outra metodologia que pode ser empregada na medição da fase do evento de reflexão foi proposta. Foi observado que a fase instantânea do traço sísmico no ponto coincidente com o máximo do envelope representa a fase da wavelet presente na janela analisada. Este método apresenta restrições de utilização, os eventos de reflexão precisam possuir uma distância em tempo considerável entre si, como apontado em Perz, Sacchi \& O' Byrne (2004). Entretanto em situações com as características necessárias esta é uma medida mais rápida.

Neste trabalho são apresentados resultados para dados sintéticos e reais de correção da wavelet de uma determinada reflexão para a fase zero. Para isto foram verificadas duas formas diferentes de medição do ângulo de correção. Os resultados foram avaliados quanto à melhoria no empilhamento da seção e quanto à precisão da estimativa da fase de correção.

\section{Metodologia}

As duas metodologias de medição da fase das wavelets (através da fase instantânea e maximização do kurtosis) são utilizadas com o intuito de corrigir as fases das wavelets de modo que se aproximem ao máximo da fase zero. Para a aplicação desta correção é realizada a rotação por uma fase constante que no domínio do tempo é descrita através da equação:

$$
y_{\text {rot }}=y(t) \cos \phi+H[y(t)] \operatorname{sen} \phi,
$$

onde $y$ representa o traço sísmico, $\phi$ é o ângulo de rotação e $H$ representa a transformada de Hilbert. 
Ambas as metodologias de estimativa de fase possuem problemas para wavelets parcialmente sobrepostas. E o efeito de mudança de fase para reflexões acima do ângulo crítico produz wavelets de fases diferentes para diferentes reflexões observadas em um mesmo traço.

Levy \& Oldenburg (1987) apresentam um exemplo de uma wavelet de fase zero convolvida com uma função refletividade dipolar resultando em um pulso semelhante a uma wavelet rotacionada de 90 graus. Neste caso, a fase estimada é 90 graus e a fase da wavelet original é perdida. Mesmo metodologias como a utilizada por van der Baan (2008), aplicando a rotação em diferentes janelas, ou a aplicada por van der Baan \& Fomel (2009), utilizando o kurtosis como um atributo sísmico local, podem apresentar problemas.

Para a estimativa através da fase instantânea do traço também foi mostrado por Perz, Sacchi \& O' Byrne (2004) que o erro na estimativa aumenta conforme as reflexões se aproximam. Por este motivo aqui foram considerados casos onde as reflexões são bem definidas e podem ser separadas.

\section{Estimativa através da fase instantânea}

Para wavelets que possuem fase constante e espectro de amplitude similar a uma função caixa é válida a propriedade de que a fase instantânea (eq. 2) no ponto correspondente ao máximo do envelope da wavelet (eq. 3) coincide com a fase obtida através da transformada de Fourier da wavelet.

$$
\begin{gathered}
\phi(t)=\tan ^{-1}\left(\frac{H[y(t)]}{y(t)}\right) \\
e(t)=\sqrt{(H[y(t)])^{2}+(y(t))^{2}}
\end{gathered}
$$

Desta forma, para a estimativa da fase é selecionado o trecho do traço que contém um evento de reflexão bem definido e são calculados o envelope e a fase instantânea nesta janela. Com isto, é obtido o ponto correspondente ao máximo do envelope e neste ponto é tomado o valor de fase.

\section{Estimativa por maximização do kurtosis}

O kurtosis, ou norma varimax, de uma série temporal discreta com $n$ amostras, pode ser descrito através da equação:

$$
V_{i}=n \frac{\sum_{i} y_{i}^{4}}{\left(\sum_{i} y_{i}^{2}\right)^{2}}-3
$$

O kurtosis do traço sísmico deve possuir valor mais alto quando a wavelet contida no traço possuir fase zero.

A busca pelo ângulo correto pode ser realizada de forma exaustiva. Através da equação (1) a parte do traço que contém a wavelet analisada é rotacionada por fases de $0^{\circ}$ a $180^{\circ}$, a fase que retornar uma wavelet com um valor de kurtosis mais alto é a fase correta.
O resultado de aplicação da rotação com o kurtosis possui uma ambiguidade quanto à polaridade da fase, fazendo com que o pulso obtido seja positivo ou negativo aleatoriamente. Neste trabalho todos os pulsos foram corrigidos para que a amplitude máxima fosse positiva. Para isto os pulsos de amplitude máxima negativa foram multiplicados por menos um e foi somado um valor de $180^{\circ}$ na fase estimada.

\section{Resultados}

\section{Dados Sintéticos}

Para a avaliação da metodologia estudada foi construído um sismograma sintético assumindo camadas planas com base no modelo intervalar da Tabela 1. Foi gerado um sismograma para um conjunto CMP com afastamentos variando de 1 a 100 metros e foi adicionado ruído aleatório na razão sinal/ruído igual a 40 (Figura 1). Esses dados permitem a avaliação da metodologia adequada para a identificação da fase das wavelets no caso de sobreposição de reflexões.

Tabela 1 - Modelo intervalar utilizado para gerar os dados sintéticos.

\begin{tabular}{ccccc}
\hline Camada & $\mathrm{H}(\mathrm{m})$ & $\mathrm{Vp}(\mathrm{m} / \mathrm{s})$ & $\mathrm{Vs}(\mathrm{m} / \mathrm{s})$ & $\rho\left(\mathrm{g} / \mathrm{cm}^{3}\right)$ \\
1 & 5 & 370 & 112 & 1,53 \\
2 & 25 & 1650 & 312 & 1,91 \\
3 & - & 4200 & 1680 & 2,54 \\
\hline
\end{tabular}

A análise de velocidades foi realizada pela equação da hipérbole deslocada, derivada por Malovichko (1978) e descrita por Castle (1984) para uma Terra de camadas horizontais. Após a análise de velocidades foi realizado silenciamento dos dados para a separação dos eventos de reflexão e correção NMO através da técnica Block Move Sum (RUPERT \& CHUN, 1975). Nesta técnica é aplicado um deslocamento constante ao longo do traço, como uma correção estática. Os dois eventos corrigidos de NMO separadamente foram somados e apresentados na Figura 2. 


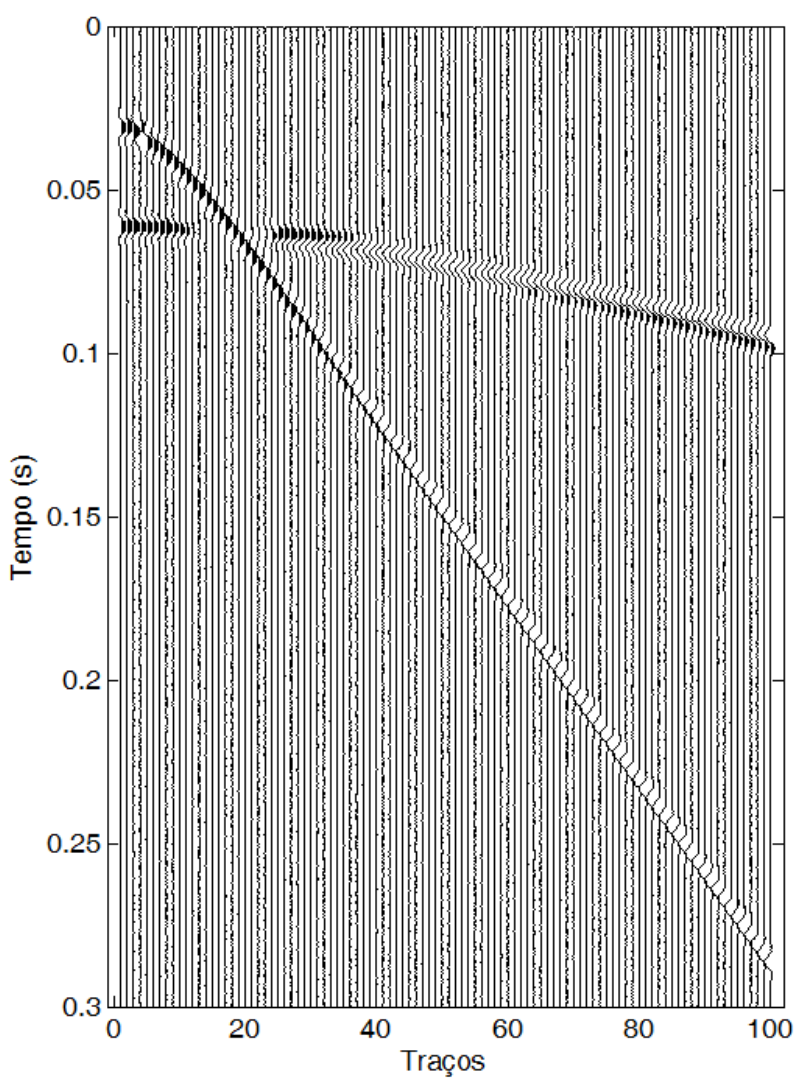

Figura 1 - Conjunto CMP sintético.

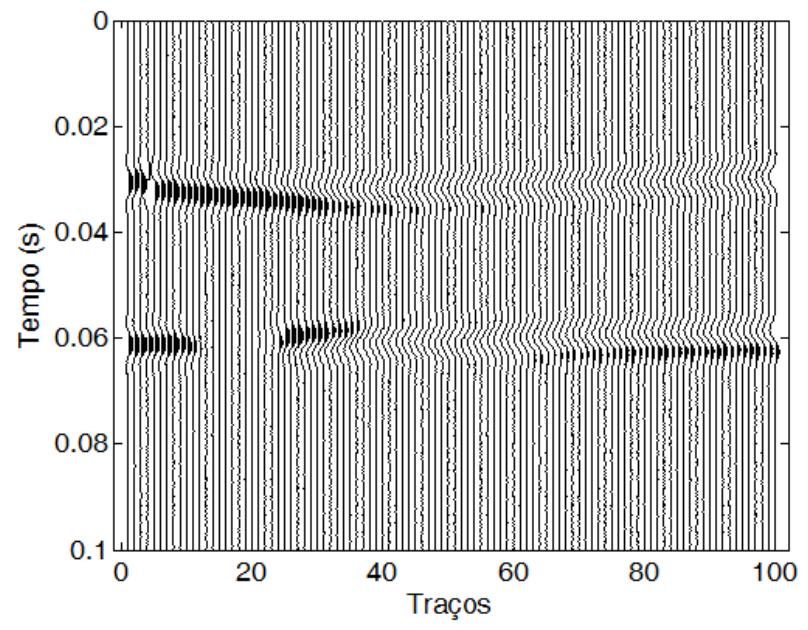

Figura 2 - Conjunto CMP sintético, corrigido de NMO pelo método de Block Move Sum.

A medição de fase foi realizada separadamente sobre cada evento de reflexão e foi aplicada uma suavização nas fases medidas para que fosse mantida a coerência entre os eventos de cada traço mesmo na presença de ruído. Para comparação dos resultados foi realizado o empilhamento dos traços da Figura 2 e dos mesmos dados com as fases corrigidas (Figura 3).

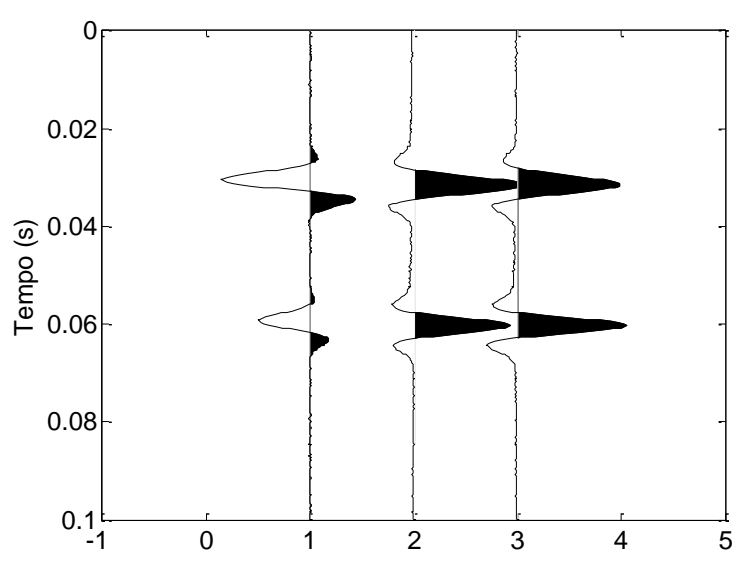

Figura 3 - Resultado do empilhamento: (traço 1) sismograma sem correção de fase; (traço 2) após a correção de fase utilizando as fases medidas através da fase instantânea; e (traço 3) após a correção utilizando o kurtosis.

Além da melhoria no empilhamento, cada uma das normas foi avaliada quanto aos resultados na medição do ângulo de correção. Nas figuras 4 e 5 são mostrados os ângulos de correção para as reflexões 1 e 2 respectivamente.

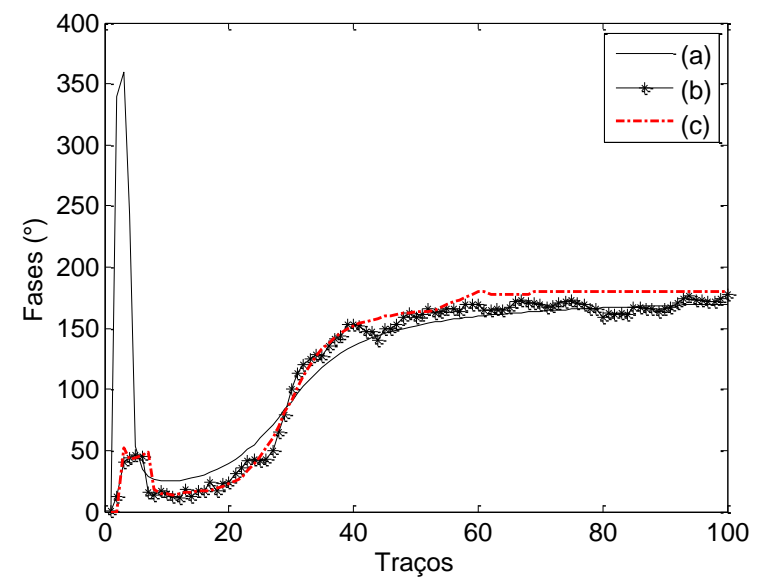

Figura 4 - Resultado da estimativa dos ângulos de correção para o primeiro evento de reflexão do sismograma da Figura 1. Sendo: (a) os ângulos originais, (b) os ângulos medidos a partir da fase instantânea e (c) os ângulos medidos através da maximização do kurtosis. 


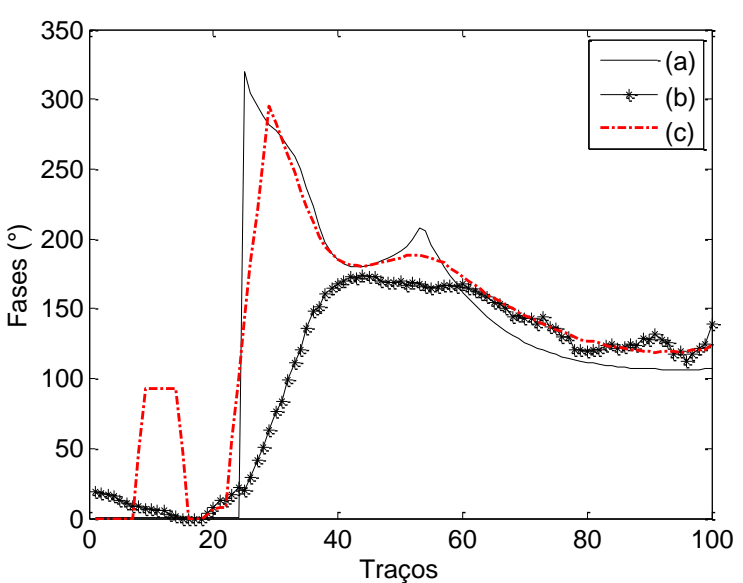

Figura 5 - Resultado da estimativa dos ângulos de correção para o segundo evento de reflexão do sismograma da Figura 1. Sendo: (a) os ângulos originais, (b) os ângulos medidos a partir da fase instantânea e (c) os ângulos medidos através da maximização do kurtosis.

\section{Dados Reais}

Para a aplicação em dados reais foram utilizados dois conjuntos CMP de uma seção sísmica adquirida em meio urbano, no terreno do Instituto de Física da USP (Mendes, 2011). A aquisição foi realizada com a técnica Mini-Sosie (BARBIER et al., 1976) utilizando um compactador de solos como fonte de energia sísmica. Desconsiderando 0 efeito da mudança de fase decorrente de reflexões acima do ângulo crítico, este tipo de fonte deveria produzir wavelets de fase zero no sismograma.

Os dados foram adquiridos para afastamentos acima de $51 \mathrm{~m}$, segundo uma técnica CMP não convencional, na qual a janela de afastamentos não é a mesma para todos os sismogramas CMP ao longo da linha. Isto faz com que a variação de fase da wavelet não seja a mesma em todos os sismogramas. Foram avaliados dois conjuntos CMP, selecionados de forma a verificar as janelas de afastamentos mais distintas: o CMP de número 24 que possui janela de afastamentos de $51 \mathrm{~m}$ a $97 \mathrm{~m}$ e o CMP de número $48 \mathrm{com}$ janela de afastamentos de $75 \mathrm{~m}$ a $121 \mathrm{~m}$.

Foi possível observar um único evento de reflexão interpretado como sendo do topo rochoso. $O$ processamento dos conjuntos CMP, após filtragem de frequência, seguiu uma sequência próxima à aplicada aos dados sintéticos. Por se tratar de um único evento de reflexão foi possível estimar os tempos de trânsito através do ponto de máximo do envelope de cada traço. Com isto, a curva da hipérbole deslocada foi ajustada aos valores de tempo (Figura 6). Em seguida, foi realizada a correção NMO dos dois conjuntos com a técnica Block Move Sum e a correção do efeito de estática observado. Para a correção estática, os eventos foram reposicionados de forma que o máximo do envelope de cada evento ficasse ajustado ao valor médio em tempo dos máximos dos envelopes do evento de reflexão observado em cada traço. Sobre esses dados foi realizada a correção de fase pelas duas metodologias avaliadas (Figura 7).
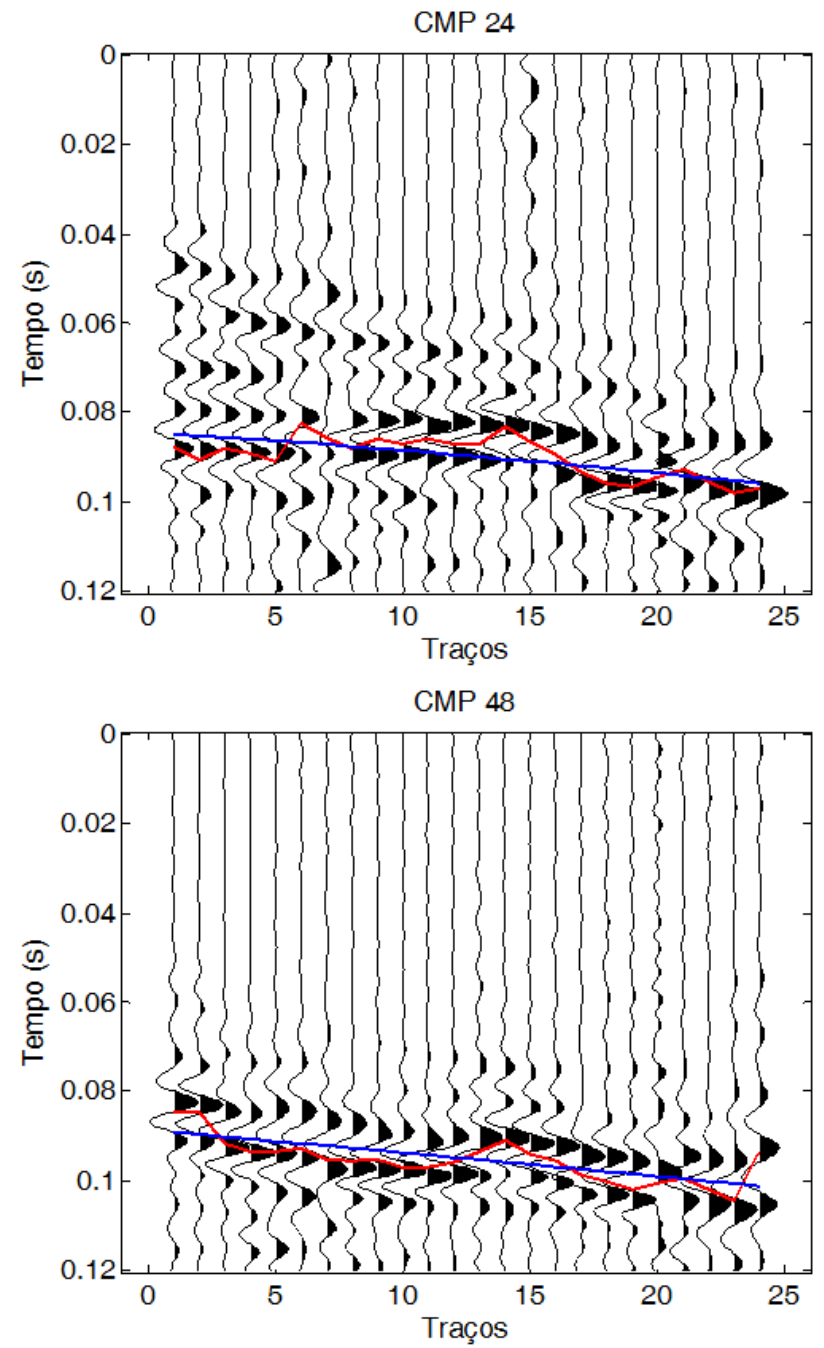

Figura 6 - Conjuntos CMP avaliados. A linha vermelha dos sismogramas é o máximo do envelope do traço na região do evento de reflexão e linha a azul é a curva ajusta os pontos da linha vermelha.

Os sismogramas da Figura 7 foram empilhados para uma melhor comparação dos resultados. Na Figura 8 também foram expressos os resultados do empilhamento convencional (análise de velocidades baseada na equação da hipérbole e correção de NMO convencional).

\section{Discussão e Conclusões}

Neste trabalho foram avaliadas duas técnicas de medição de fase das wavelets presentes nos traços sísmicos para posterior correção das mesmas. Os métodos foram testados sobre dados de sísmica em uma escala rasa, adquiridos acima do ângulo crítico de incidência.

A correção das fases, medidas pelas duas técnicas melhorou a coerência dos sinais, resultando em um melhor empilhamento para ambos os casos, sintético (Figura 3) e real (Figura 8). 
Além disso, nos dados reais é possível notar uma maior semelhança no pulso empilhado após a correção das fases dos conjuntos CMP adquiridos em janelas de afastamentos distintas (comparar os dois CMPs da Figura 8). Ainda na Figura 8 também foi mostrado que a correção NMO por Block Move Sum gera um pulso empilhado de maior frequência e mais coerente entre os diferentes conjuntos CMP.

Verificou-se nos dados sintéticos e reais que a técnica de medição da fase instantânea se mostrou mais sensível ao ruído, o qual afeta o cálculo de envelope do sinal via transformada de Hilbert. Nos dados reais, o resultado do empilhamento após a correção de fase foi superior para a técnica que utiliza o kurtosis, como pode ser observado nas Figuras 7 e 8.

Por fim, conclui-se que o procedimento de correção de fase constante utilizando o kurtosis como critério é o mais indicado, tanto no processamento dos dados melhorando o empilhamento, quanto na determinação do valor da defasagem da wavelet.

As principais perspectivas de uso da metodologia avaliada são: em dados de sísmica rasa, que frequentemente são adquiridos acima do ângulo crítico de incidência, situação em que a mudança de fase com o afastamento é sempre presente; e a possibilidade de extração dos valores de fase dos pulsos refletidos, a serem utilizados como informação adicional para inversão dos parâmetros dos meios.

\section{Agradecimentos}

Allan Segovia Spadini agradece a Capes pela bolsa de mestrado.

\section{Referências}

Aki, K. \& Richards, P. G., 1980. Quantitative seismology, Theory and methods, v. 1: W. H. Freeman and Co., p.557

Arons, A. B., \& Yennie, D. R., 1950. Phase distortion of acoustic pulses obliquely reflected form a medium of higher sound velocity. J. Ac. Soc. Am., v.22, p. 231-237.

Barbier, M. G. 1983. The Mini-Sosie Method. International Human Resources Development, p. 90, Boston.

Berkhout, A. J., 1977. Least-Squares Inverse Filtering and Wavelet Deconvolution. Geophysics, v.42 p. 13691383.

Bodine, J. H., 1984. Waveform Analysis With Seismic Attributes. SEG Annual Meeting. p. 5.
Castle, R. J. 1994. A theory of normal moveout. Geophysics, v. 59, n. 06, p.983-999.

Diogo, L. A., Le Diagon, F. M. \& Prado, R. L., 2004. Bedrock imaging using post-critical shallow reflection data. Journal of applied Geophysics, v. 57, p. 1-9.

Levy, S. \& Oldenburg, D. W. 1987. Automatic phase correction of common-midpoint stacked data. Geophysics, v. 52, p. 51-59.

Longbottom, J., Walden, A. T. \& White, R. E. 1988. Principles and application of maximum kurtosis phase estimation. Geophysical Prospecting, v. 36, p. 115-138, 1988

Malovichko, A. A. 1978. A new representation of the traveltime curve of reflected waves in horizontally layered media. Applie Geophysics, n. 91, p. 47-53 (in Russian).

Mendes, S. C. 2011. Levantamento de reflexão sísmica rasa empregando fonte Mini-Sosie em afastamentos longos. Trabalho de Graduação. Instituto de Astronomia, Geofísica e Ciências Atmosféricas. Universidade de São Paulo. P. 39

Perz, M., Sacchi, M. D. \& O'Byrne A. 2004. Instantaneous phase and the detection of lateral wavelet stability. The Leading Edge, p. 639-643.

Pullan, S. E. \& Hunter, J. A. 1985. Seismic model studies of the overburden-bedrock reflection. Geophysics, v. 50, p. 1684-1688.

Rupert, G. B. \& Chun, J. H. 1975. The block move sum normal moveout correction. Geophysics, v. 40, n.01, p.1724.

Van der Baan. 2008. Time-varing wavelet estimation and Deconvolution using kurtosis maximization. Geophysics, v. 73 , p. V11-V18.

Van der Baan, M. \& Fomel, S. 2009. Nonstationary phase estimation using regularized local kurtosis maximization. Geophysics, 74, A75-A80.

White, R. E. 1988. Maximum kurtosis phase correction. Geophysical Journal, v. 95, p.371-389.

Wiggins, R. A. 1978. Minimum Entropy Deconvolution. Geoexploration, v. 16, p.21-35, Amsterdam. 
(a)

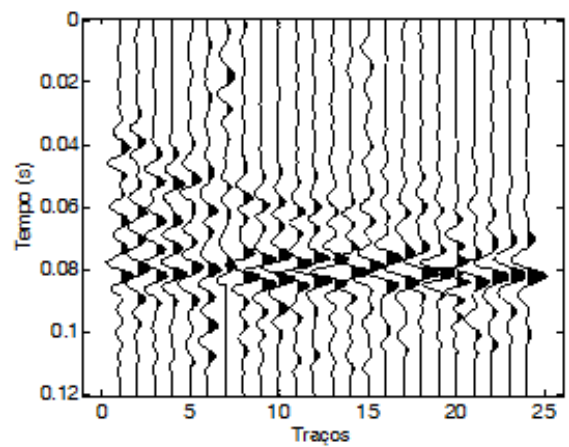

(d)

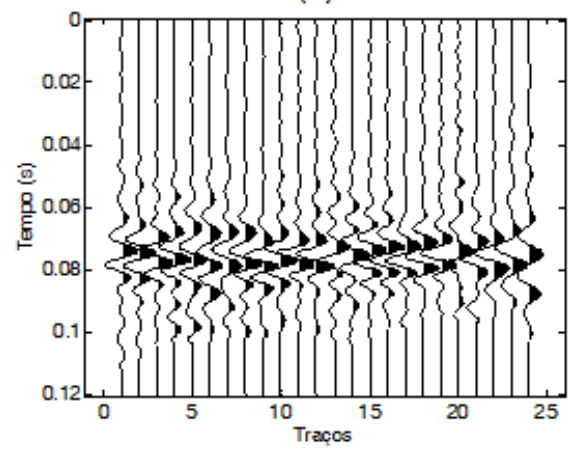

(b)

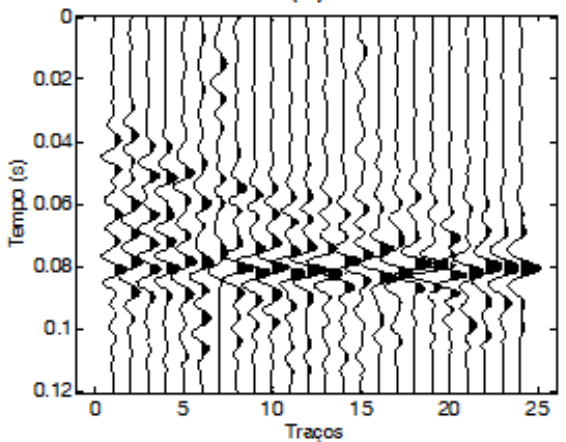

(e)

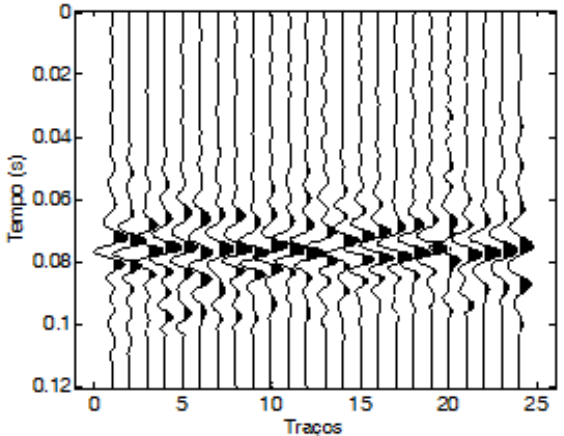

(c)

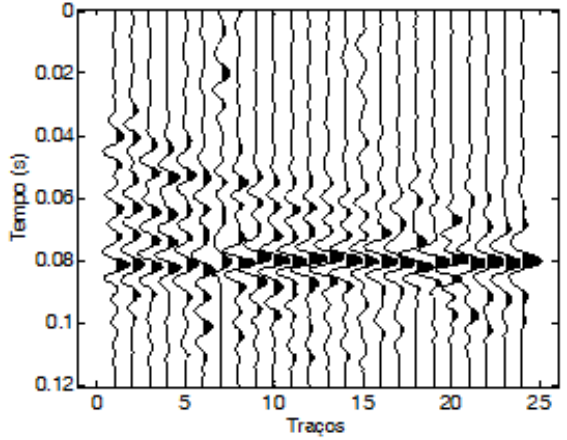

(f)

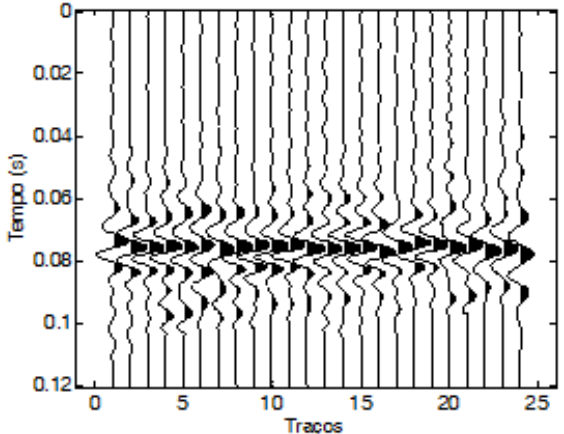

Figura 7 - Resultado da correção de NMO pela técnica Block Move Sum dos CMPs 24 e 48 respectivamente. Sendo: (conjuntos (a) e (d)) seção sem correção de fase; (conjuntos (b) e (e)) da seção após a correção de fase utilizando as fases medidas através da fase instantânea; e (conjuntos (c) e (f)) da seção após a correção utilizando o kurtosis.
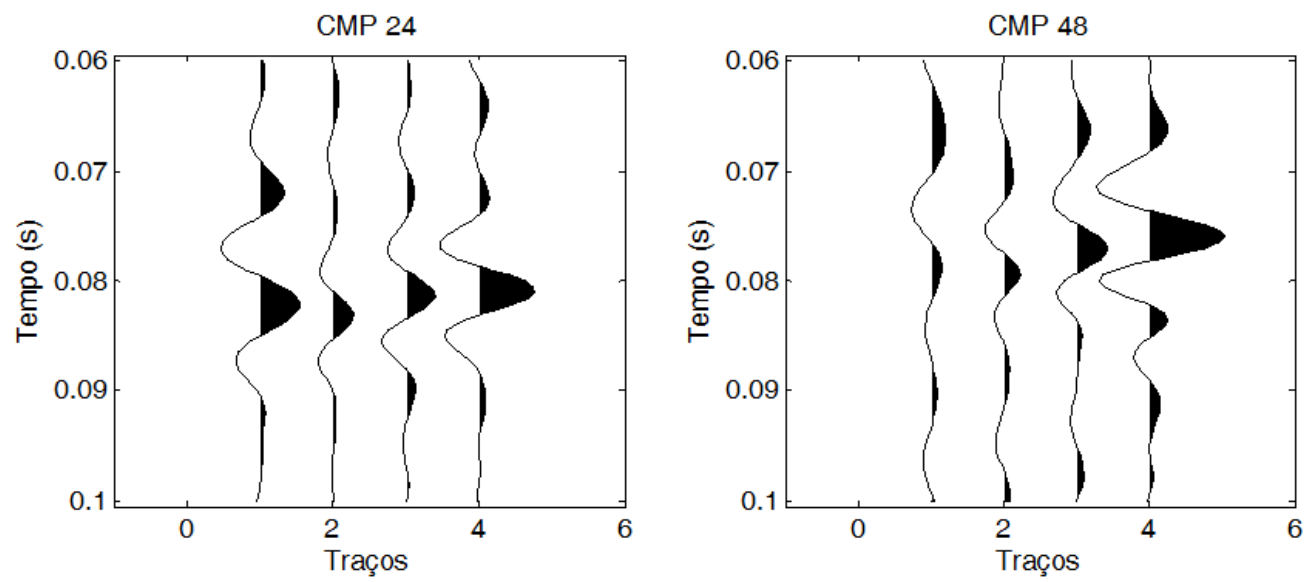

Figura 8 - Resultado do empilhamento dos CMPs 24 e 48. Sendo: (traço 1) resultado para a seção processada de forma convencional; (traço 2) seção sem correção de fase; (traço 3) da seção após a correção de fase utilizando a fases medidas através da fase instantânea; e (traço 4) da seção após a correção utilizando o kurtosis. 